\title{
SURGICAL PATHOLOGY OF HYDATID CYSTS OF THE LUNG
}

\author{
By C. J. Officer Brown, M.D., F.R.C.S., F.R.A.C.S. \\ Consulting Thoracic Surgeon, Alfred Hospital, Melbourne
}

A hydatid cyst in the lung starts with the lodgment of the hexacanth embryo of Echinococcus granulosus in the interstitial tissue of the lung, and a small, progressively growing cyst develops. The rate of growth varies but quite large cysts may be seen in children of two or three years old, or in immigrants, who have only been in Australia for a short period and who had clear X-rays on arrival.

Around the cyst a fibrous adventitial capsule is formed and, as it enlarges, it compresses alveoli and impinges on blood vessels and bronchi. Blood vessels are eroded and this explains the frequency of haemoptyses as a symptom of hydatid disease of the lungs. Bronchi are also eroded and open into the adventitiae and, consequently, when the cyst is collapsed or removed there is almost always a bronchial communication with its bed and, when rupture occurs into the pleural cavity, a pneumothorax is almost certain to follow and may develop tension. In my experience every cyst has been somewhere in contact with visceral pleura. This may be on the diaphragmatic surface or the mediastinal surface or in a fissure, but, in consequence of this relationship, they can all be removed without cutting air-containing lung. Because the costal surface is so much greater than the other surfaces of the lung, the majority of cysts will be found somewhere in contact with the chest wall.

During the last 20 years ${ }_{1} 2$ patients have been operated upon in the Alfred Hospital for hydatid disease of the lungs and pleura: 173 operations were done to remove 186 cysts, or their results, from the thoracic cavity and seven from the liver. All the operations were carried out by myself or Mr. Kenneth Morris, who has worked with me in the Surgical Thoracic Unit for the last eight years. No patient died as a result of operation. In six patients with post-hydatid cavities and in one with a large degenerated calcified cyst the diagnosis was not confirmed by the recovery of hydatid material at operation, but in all these patients the past history of causative hydatid disease was unequivocal, in every other patient hydatid disease was proved at operation.
In Table $\mathrm{I}$, the total number of cysts and patients has been listed. In 68 patients all the cysts were simple and unruptured. The total of such cysts removed was 85 , but some of these occurred in the lungs of 66 patients of Group 2, who all had at least one cyst ruptured into a bronchus. In these 66 patients, a total of 76 cysts had undergone intrabronchial rupture. In seven patients pulmonary cysts were complicated by intrapleural rupture at the time of operation, and in three patients liver cysts had ruptured into the pleura, and in one a liver cyst had ruptured into a bronchus. Four patients had extrapleural cysts in the chest wall and one of these had extended into the spinal canal, causing paraplegia, and one had a degenerated cyst in the diaphragm. Two patients were seen for the first time with recurrent pleural cysts, and three patients, from whom simple cysts had been removed, returned for a second operation for recurrent cysts of the pleura. No patient has been included twice in Table $\mathrm{I}$, but every cyst removed has been tabulated. Nine patients had bilateral cysts and two operations were done in eight of them. In the last patient, two cysts in the right lung and one in the left were removed at the same operation.

Although a cyst may remain simple for many years and may grow to occupy the greater part of a pleural cavity, the majority sooner or later rupture into a bronchus, and in this series more than half the cysts had ruptured in this way before they were recognized. Usually the rupture floods the bronchial tree with fluid, and blood and fluid are coughed up with great distress to the patient, but it may be quite silent and many patients, who present with collapsed cysts, have no idea when the fluid was evacuated. Usually the membrane is retained within the adventitial cavity but occasionally it is evacuated in one piece and patients have been suffocated by impaction of the membrane in the larynx. Immediately after rupture an X-ray picture of the lungs shows a typical appearance. The cyst is represented by an air-containing space with a fluid level and membrane showing irregu- 
TABLE I

Hyidatid Disease of Lungs ani Pifera

Summary of 152 Patients With no Mortality

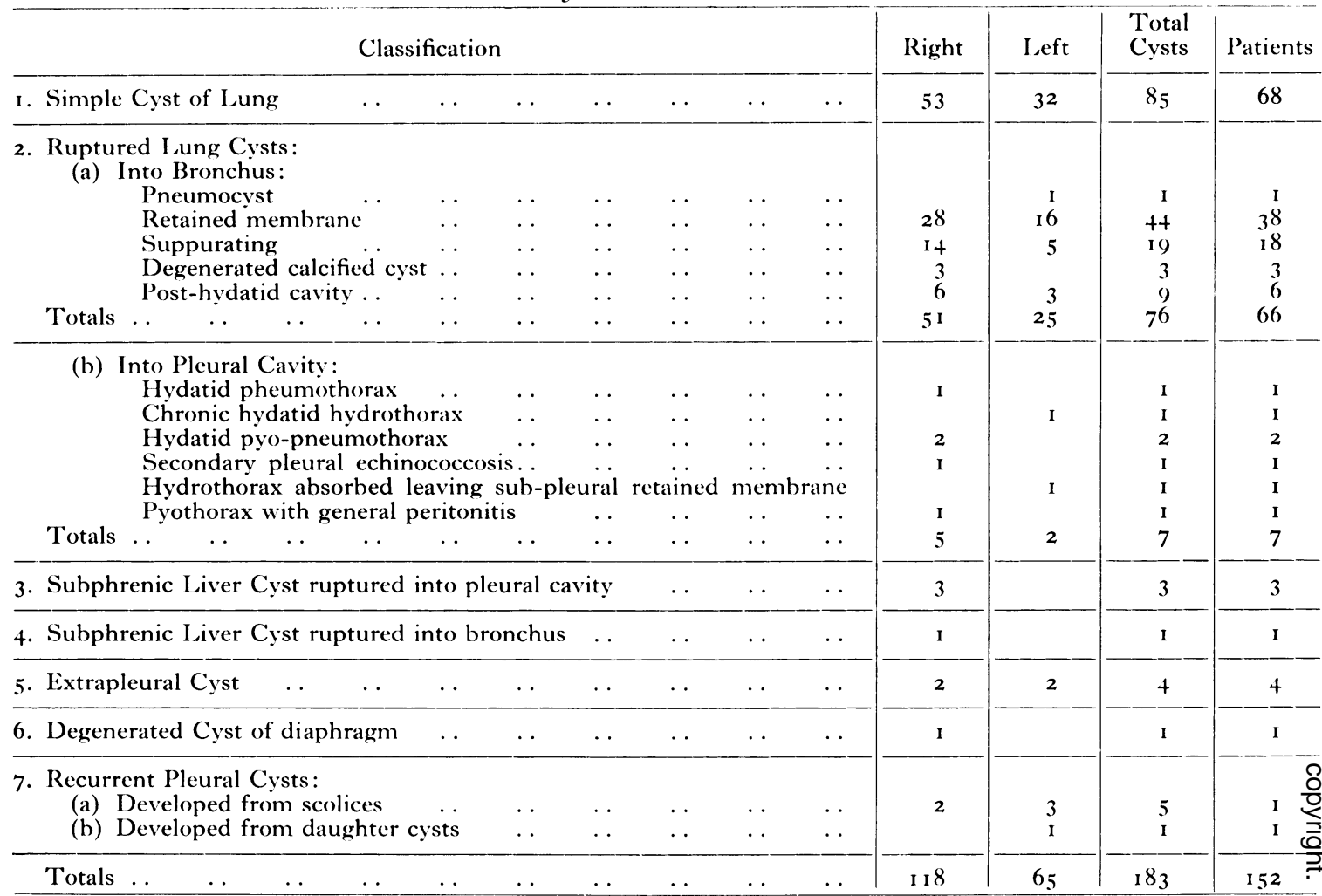

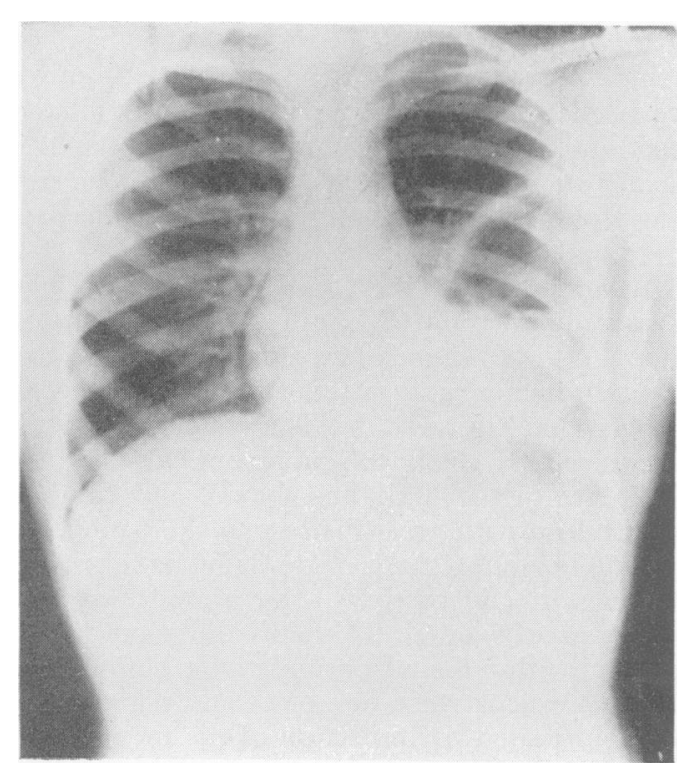

Fis: 1.-Cyst of the lingula, after intrabronchial rupture, showing air-containing pneumocyst with fluid level and crumpled membrane floating above the fluid. larly above the fluid (Fig. I). Air may be trapped in the space and the tension cyst may require urgent decompression. If nothing is done at this stage, the fluid and air usually disappear and the adventitia shrinks down on the crumpling membrane and an opaque shadow, much smaller than the original cyst, remains in the X-ray. Unless the history of rupture is clean-cut or immunological tests are positive, diagnosis from other lumps in the lung is often impossible. This stage may persist for many years causing no symptoms. Occasionally daughter cysts will develop amongst the crumpling membrane and this is the only circumstance in which $I$ have seen them in lung cysts. 'Their rarity suggests that, in most cases, the upset of rupture and evacuation devitalizes the cyst. Degeneration of the membrane and calcification may occur and again, I believe, this is the only circumstance in which calcification is seen in hydatid cysts in the lung.

Suppuration may complicate the picture after rupture and may commence either in the pneumocyst stage or in the stage of retained membrane. Usually it is an indolent infection contained within the adventitia, resulting in cough with expectora- 


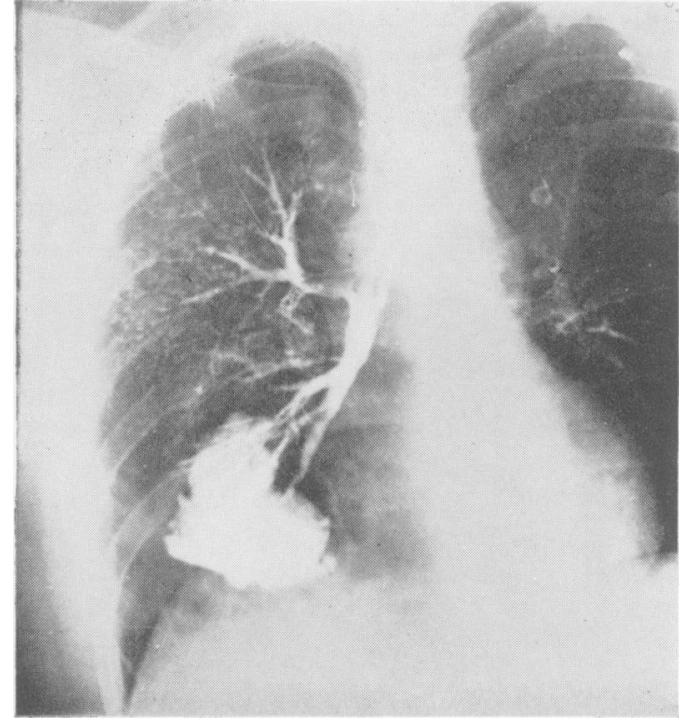

FIG. 2.-Bronchogram showing post-hyddatid cavity left after the removal of a large suppurating cyst. Note several bronchi running into cavity. Patient was suffering from frequent haemoptyses.

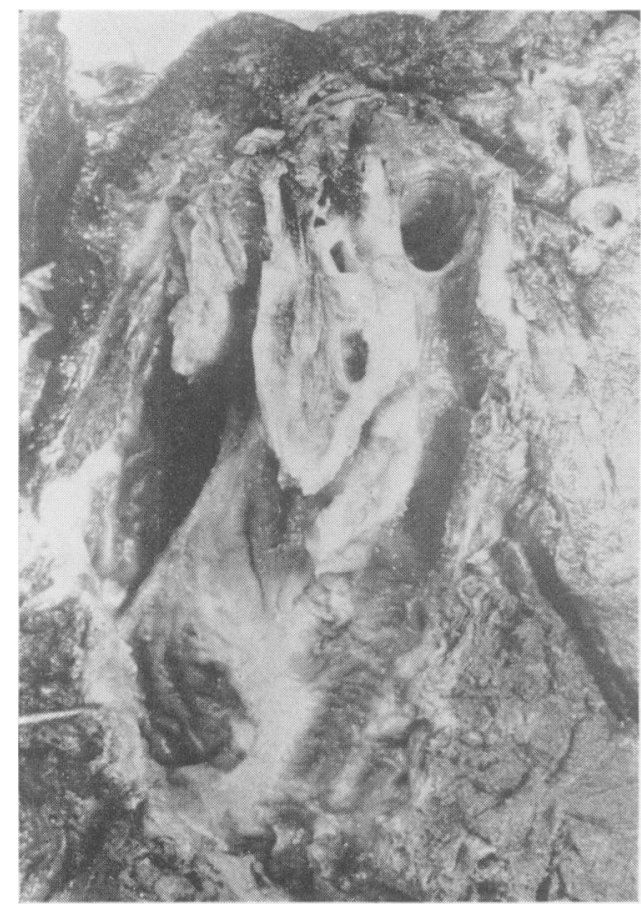

Fic: + - Post-hydatid cavity laid open.

tion, and the membrane may gradually disintegrate and be coughed up, but it is surprising how normal

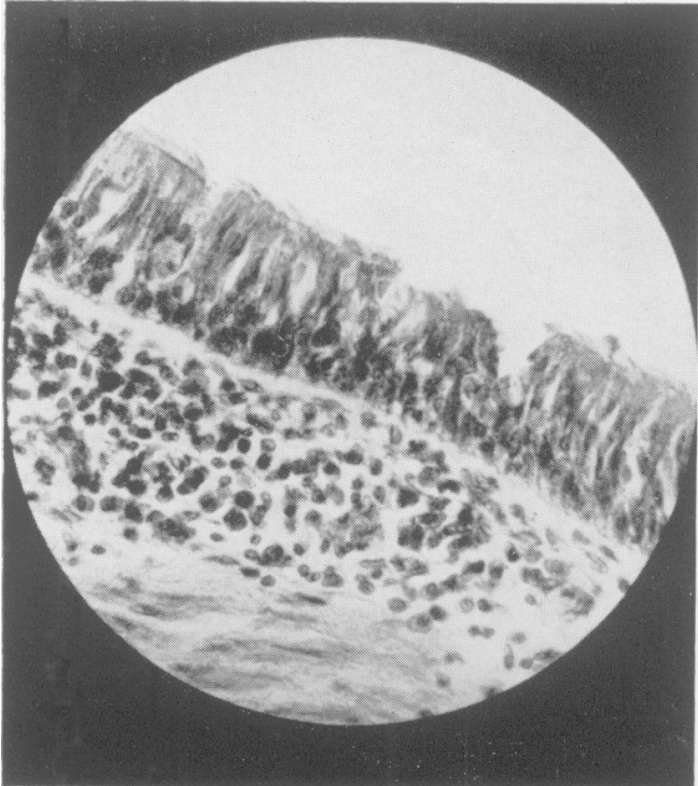

Fig. 3.-Respiratory epithelium lining post-hydatid cavity.

a membrane may remain, even after it has been bathed in pus for several years.

After the final evacuation of the cyst, the adventitial cavity shrinks down and may obliterate, but in many cases a pocket, demonstrable by bronchography, persists and this is more likely to be seen when evacuation after rupture has been delayed and accompanied by suppuration (Fig. 2). 'These cavities become lined by respiratory-type epithelium (Fig. 3), although there may be some areas without epithelium, and some areas are covered with squamous cells, and they have several bronchi opening into them (Fig. 4). They may be quite symptomless or may cause repeated haemoptyses or may become intermittently or chronically infected.

The result after operative removal of a cyst is the same as that following spontaneous rupture, and a post-hydatid cavity may persist but, if a cyst that is not suppurating is cleanly removed and there is no prolonged infection in the space after operation, troublesome post-hydatid cavities are rarely seen.

In some cases suppuration extends beyond the adventitial space, and some segments or a lobe may develop the changes of chronic pulmonary suppuration and bronchiectasis similar to those seen with other retained foreign bodies (Fig. 5).

In a smaller proportion of patients the cyst ruptures into the pleural cavity. Intrabronchial spill with cough and expectoration may occur at the 


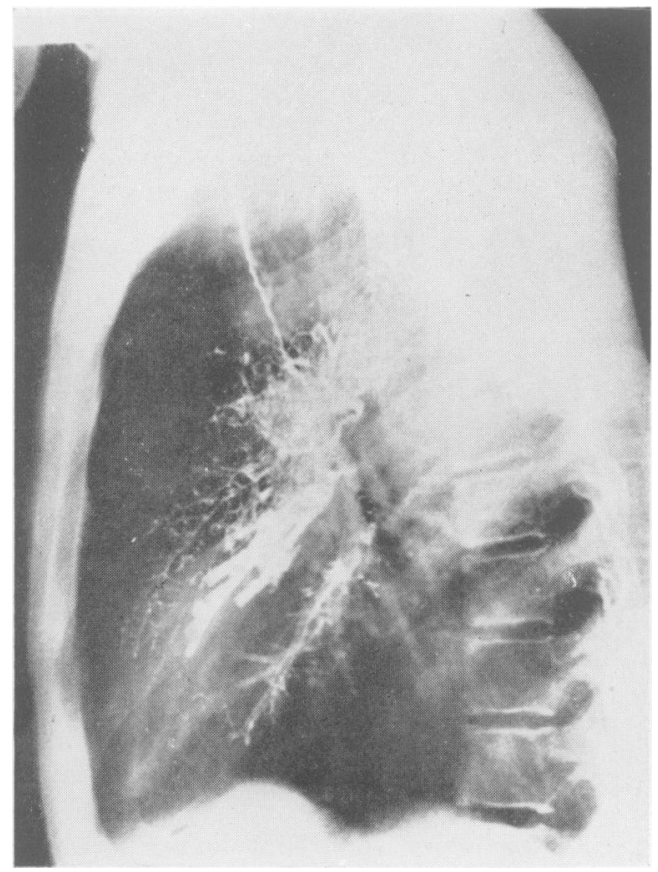

Fig. 5.-Bronchiectasis following removal of a suppurating cyst in the lingula. Patient suffered from repeated haemoptyses.

same time, or the onset may be similar to that of other spontaneous pneumothoraces. The fluid empties into the pleural cavity and air escapes, a pneumothorax develops and may develop tension. The membrane may remain within the adventitia or may be expelled into the pleural cavity. In either case the rupture may seal and the air and fluid be absorbed. If the membrane is still within the adventitia, when the lung re-expands, the picture is that of retained membrane following intrabronchial rupture, but, in addition, secondary pleural cysts may develop from spilled scolices.

In two of my patients, the air leak closed after the membrane had escaped into the pleural cavity and, in one, a chronic hydrothorax without fistula persisted until the membrane was removed and the lung decorticated. In the other the lung reexpanded and the membrane was encapsulated in a small pleural pocket.

Suppuration may occur and the patient may present with a pyopneumothorax. One of my patients was seen after intrabronchial rupture of his cyst with a typical pneumocyst. Operation was advised but he elected to delay and hoped for a spontaneous cure. Six weeks later he developed peritonitis. No cause for the peritonitis was found in his abdomen at laparotomy, but it was then recognized that he had a large pyothorax and

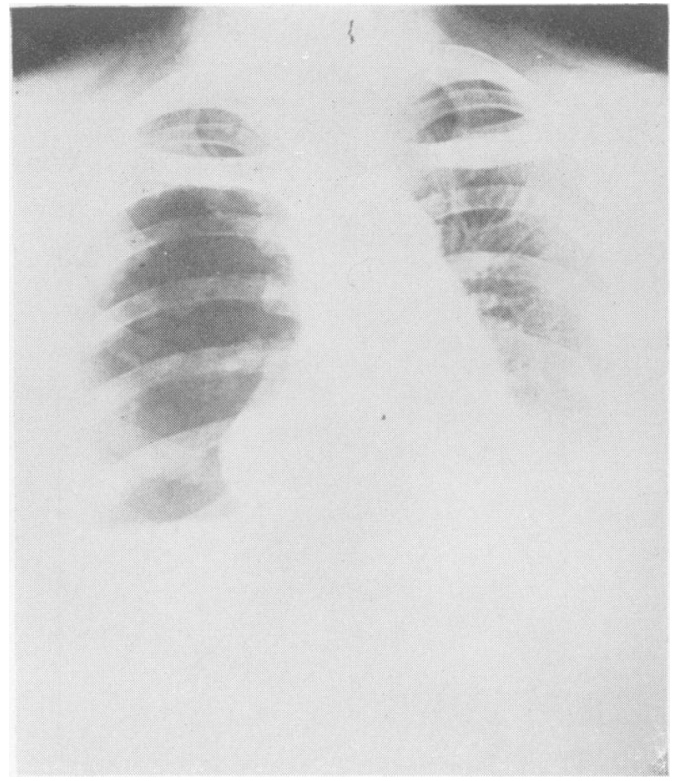

Fis. 6.-Rupture of cyst into pleural cavity with hydatid pneumothorax and secondary pleural echinococcus. Note multiple small cysts studded over parietal pleura. Rupture had occurred six months earlier.

prompt recovery followed evacuation of the pyothorax and removal of his cyst.

Secondary pleural Echinococcus (Fig. 6) results from the development of cysts in the pleural cavity following the spilling of scolices. It is rare and follows only a small proportion of intrapleural ruptures. Although millions of scolices are spilled in such a rupture, only a very few, if any, secondary cysts develop and even where scolices were recklessly spilled at operation, as was done by many Australian surgeons before the development of modern techniques, very few patients presented with recurrent pleural cysts.

Because the lung cysts which rupture into the pleural cavity are always simple without daughter cysts, the problem of intrapleural spilling is quite different to that of intraperitoneal spilling from liver cysts containing daughter cysts and brood capsules in large numbers. In one patient of my series, operated on primarily by another surgeon, daughter cysts were present, presumably in a previously ruptured and collapsed cyst, and it was assumed that the escape of one or more of these at operation was responsible for the development of a localized posterior pleural pocket stuffed with small cysts and debris, which I evacuated i 5 years later.

Liver cysts may rupture through the diaphragm into the pleural cavity and may result in a hydrothorax which will usually contain bile and daughter 
cysts. Where the lung is adherent at the time of rupture, a broncho-biliary fistula will result, and this is the commonest cause of this condition in Australia. Sometimes no bile is expectorated but, even in its absence, the expectoration of daughter cysts should always suggest a primary liver cyst with a bronchohepatic fistula, because daughter cysts are so rare in pulmonary hydatids.

Hydatid cysts may occur in any part of the body and we have seen three lying extrapleurally against the spine. Cysts in this position are important because they tend to rupture and extend extrapleurally with the production of multiple daughter cysts and, following a line of least resistance, may spread through a spinal foramen causing hydatid paraplegia, as had occurred in one of my patients.

Cysts of the diaphragm are occasionally reported and one, in a degenerated condition when it was removed at operation, is included in this series.

\section{Extrathoracic Cysts}

Four of these patients had previously been operated on for the removal of liver cysts and we removed liver cysts from seven more of them. One child had a cerebral cyst and a pelvic cyst removed, and had a cyst in the liver and one in the lung. We removed the large lung cyst, because it seemed likely to rupture, but did not operate on the liver cyst. He has since died as a result of the recurrence of the cerebral cyst. Although it is generally held that the liver is the commonest site for hydatid disease in man, Clunies Ross ${ }^{1,2}$ found that lung cysts were more common in sheep and cattle, and I suspect that the figures relating to man may be fallacious, because in the past many lung cysts were evacuated spontaneously and not diagnosed. Since the introduction of routine chest radiography, many more lung cysts have been found and quite a proportion of the cases included in this series presented with symptomless lumps, and many of them would have gone through life unrecognized if it had not been for the X-ray.

\section{REFERENCES}

1. ROSS, I. CLUNIES (1926), Med. F. Aust., 1, 96.

2. ROSS, I. CLUNIES (1929), 'Observation of the Hydatid Parasite, 'Commonwealth of Australian Council of Scientific and Industrial Research, Bulletin No. 40, Melbourne.

\title{
CARCINOMA OF THE BRONCHUS
}

\author{
Price 3s. 11d. post free
}

\section{INTRODUCTORY}

Maurice Davidson, D.M., F.R.C.P.

THE INCIDENCE AND AETIOLOGY OF PRIMARY CARCINOMA OF THE LUNG

C. E. Drew, M.V.O., F.R.C.S.

MEDICAL ASPECTS

J. Anderson, M.D., F.R.C.P.

RADIOLOGICAL ASPECTS

G. Simon, M.D., D.M.R.E., F.F.R.
UNUSUAL MANIFESTATIONS

J. Smart, M.D., F.R.C.P.

CYTOLOGICAL EXAMINATION OF THE SPUTUM AND PLEURAL EFFUSION

J. L. Pinniger, D.M., M.R.C.P.

THE SCOPE OF RADIOTHERAPY

Gwen Hilton, D.M.R.E., F.F.R.

SURGERY OF CARCINOMA OF THE BRONCHUS

L. L. Bromley, M.Chir., F.R.C.S.

Published by

THE FELLOWSHIP OF POSTGRADUATE MEDICINE

60, Portland Place, London, W.1 